\title{
Acetilação da Fibra de Bucha (Luffa cylindrica)
}

\author{
Ana L. F. S. d’Almeida, Verônica Calado, Daniel W. Barreto \\ Escola de Química, UFRJ
}

José R. M. d'Almeida

DCMM, PUC-Rio

\begin{abstract}
Resumo: Neste trabalho, foi analisado o efeito de um tratamento químico, ou seja, de acetilação de grupos, sobre a estrutura e a morfologia superficial de fibras de bucha (Luffa cylindrica) por meio de FTIR e microscopia eletrônica de varredura. Esse tratamento foi realizado tendo por objetivo aumentar a compatibilidade da bucha com as matrizes poliméricas usualmente empregadas em compósitos. Mostrou-se que o tratamento reduziu a polaridade das moléculas de celulose e removeu a camada superficial das fibras de bucha, expondo a sua estrutura fibrilar interna, com conseqüente aumento da área disponível para a adesão.
\end{abstract}

\section{Palavras-chave: Luffa cylíndrica, tratamento superficial, compósitos.}

\section{Acetylation of Loofah Fiber (Luffa Cylindrica)}

Abstract: In this work the effect of a new chemical surface treatment on the structure and surface morphology of loofah fibers (Luffa cylindrica) was analyzed, using infrared spectroscopy and scanning electron microscopy. The chemical treatment is aimed at increasing loofah compatibility with the polymeric matrices commonly used in composites. It was shown that the treatment used caused a reduction on the polarity of cellulose molecules, and removed the outer surface layer of loofah fibers, exposing their internal fibrillar structure. As a consequence the treatment promoted an increase on the surface area available to adhesion.

Keywords: Luffa cylindrica, surface treatment, composites.

\section{Introdução}

O emprego de compósitos de matriz polimérica reforçados por fibras naturais lignocelulósicas vem tendo um crescente desenvolvimento nos últimos anos ${ }^{[1-3]}$. Os grandes atrativos desses materiais incluem o fato das fibras serem biodegradáveis, serem um recurso natural renovável, terem geralmente baixo custo e produzirem menor desgaste nos equipamentos de fabricação quando comparadas com as fibras sintéticas. Dentre as principais fibras vegetais mais estudadas e empregadas em nível mundial como reforço em compósitos de matriz polimérica, destacam-se o sisal e a juta ${ }^{[4,5]}$. Na Europa, estão sendo aplicados pesados investimentos para o emprego de fibras nativas como o linho e o cânhamo ${ }^{[3]}$. Entretanto, inúmeras outras fibras vegetais mostram potencialidade de aplicação como reforço em compósitos de matriz polimérica. As fibras de bananeira, por exemplo, são abundantes, tendo uma produção mundial estimada superior a 300.000 toneladas/ ano, além de serem baratas e terem boas propriedades mecânicas ${ }^{[6,7]}$. Outras fibras que podem ser destacadas são as de coco, rami, piaçava e bucha ${ }^{[7-12]}$.

A Luffa cylindrica, vulgarmente conhecida como bucha, é uma planta subtropical, abundante na China, Japão e em outros países da Ásia, bem como em países da América Central e do Sul. Sua fruta tem uma sistema vascular que, quando seca, forma uma manta tridimensional natu$\mathrm{ral}^{[11,13]}$. A Figura 1 mostra o aspecto geral da bucha seca.

O uso de bucha como reforço em compósitos de matriz polimérica apresenta como grande vantagem a característica de manta natural contínua dessa fibra ${ }^{[11]}$. Esse aspecto estrutural confere aos compósitos de bucha uma maior tenacidade, pois as trincas são defletidas nas interfaces fibramatriz. Entretanto, mesmo com essa vantagem, os níveis de tensão obtidos para esses compósitos são baixos, devido à ruptura prematura das interfaces ${ }^{[11]}$.

A falta de boa adesão entre as fibras e as matrizes poliméricas é, na verdade, a principal desvantagem do

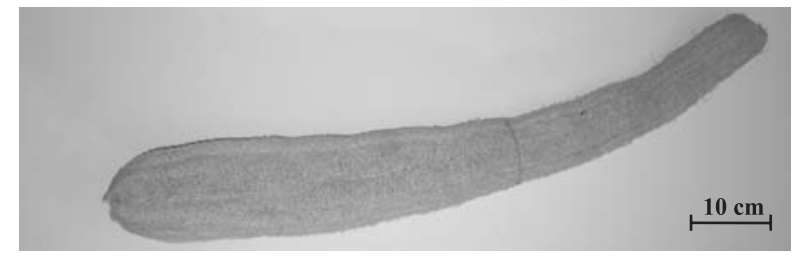

Figura 1. Fotografia da bucha, mostrando o aspecto do sistema vascular da bucha seca. 
emprego de fibras lignocelulósicas. Fibras lignocelulósicas são hidrofílicas, enquanto os polímeros usualmente utilizados como matrizes são hidrofóbicos. Desse modo, normalmente as interfaces fibra - matriz desenvolvidas são fracas, resultando em uma transferência de tensões não efetiva para as fibras. Inúmeros tratamentos físicos e químicos têm sido desenvolvidos para melhorar essa característica e, portanto, aumentar o desempenho dos compósitos fabricados com fibras naturais ${ }^{[14-18]}$. No caso da bucha, tratamentos alcalinos mostraram a possibilidade de melhorar a interface fibra-matriz, embora a melhora obtida não tenha sido significativa ${ }^{[12]}$.

Os tratamentos químicos são normalmente classificados em função do tipo da ligação carbono - oxigênio - carbono formada, destacando-se as ligações éter, éster e acetal[ ${ }^{[19]}$. Entre as diferentes possibilidades existentes para as reações de esterificação, as de acetilação apresentam grandes vantagens em termos de custo e eficiência e têm sido extensivamente usadas no tratamento de madeiras ${ }^{[19]}$.

Neste trabalho, é apresentado o desenvolvimento de um tratamento superficial ainda não aplicado em fibras de bucha, envolvendo uma reação de acetilação, que foi feita seguindo a metodologia normalmente empregada para se acetilar, usando uma solução de ácido sulfúrico, anidrido acético e ácido acético. Os mecanismos envolvidos na reação destes compostos com as fibras lignocelulósicas são apresentados e o efeito do tratamento é analisado por espectroscopia na região do infravermelho (FTIR) e por microscopia eletrônica de varredura (MEV).

\section{Experimental}

O tratamento realizado neste trabalho foi feito na bucha como recebida. As buchas usadas foram provenientes da região de São Lourenço, no sul do Estado de Minas Gerais. Antes do tratamento, as buchas foram cortadas em comprimentos de aproximadamente $14 \mathrm{~cm}$ sendo, em seguida, cortadas longitudinalmente em duas metades. $\mathrm{O}$ tratamento de acetilação teve o intuito de substituir grupos hidroxila presentes nas fibras naturais. Esse tratamento visa, assim, reduzir a polaridade da bucha, pois os grupos hidroxila presentes na celulose e, também, na hemicelulose e a na lignina são os responsáveis pelo caráter polar das fibras lignocelulósicas. De fato, os principais grupos funcionais susceptíveis à acetilação são as hidroxilas presentes na celulose, na hemicelulose e até na lignina - nesse caso a acetilação das hidroxilas fenólicas é um pouco mais difícil, mas pode ocorrer. Como a acetilação nas condições em que é realizada neste trabalho não é uma reação quimio, nem régioespecífica, acreditamos que, a princípio, todas as hidroxilas presentes podem ser atacadas. Outros grupos funcionais porventura presentes e passíveis de acetilação não são significativos em fibras lignocelulósicas.

Na Figura 2 está mostrado o esquema da acetilação parcial da celulose. Deve-se destacar que os outros principais componentes presentes nas fibras lignocelulósicas - a hemicelulose e a lignina - podem também ser acetilados.

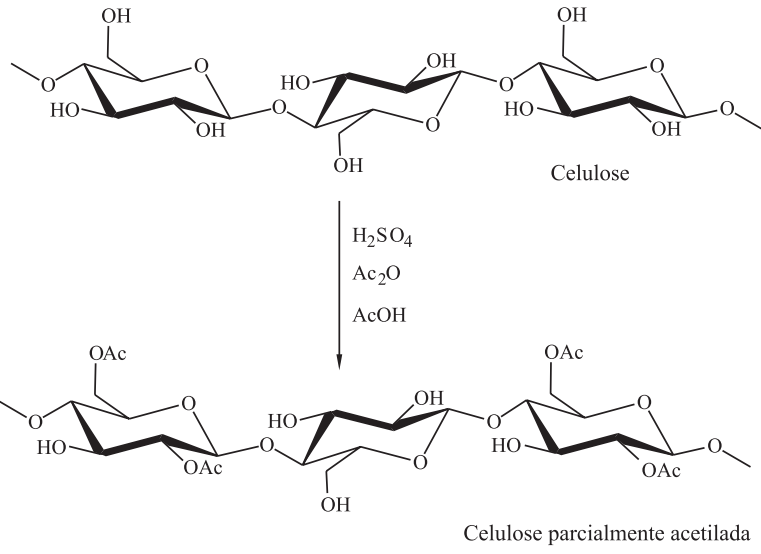

Figura 2. Esquema da acetilação parcial da celulose

As fibras cortadas, conforme descrito acima, foram imersas em uma solução de anidrido acético e ácido acético (1,5:1,0 em massa). Nessa mistura, foram adicionadas como catalisador 20 gotas de ácido sulfúrico para cada $500 \mathrm{ml} \mathrm{de}$ solução. As fibras suspensas na solução reagente foram submetidas por $3 \mathrm{~h}$ a banho em ultrasom, permanecendo posteriormente por mais $24 \mathrm{~h}$ em repouso na solução. Foram então removidas, lavadas em água corrente e colocadas por mais $24 \mathrm{~h}$ em água. Essa última etapa de lavagem e repouso foi repetida por três vezes, até que o $\mathrm{pH}$ da solução estivesse em torno de 5. As fibras foram então removidas da água e secas em estufa, a $60^{\circ} \mathrm{C}$, até peso constante.

A análise por espectroscopia na região do infravermelho foi realizada na região entre 400 e $4000 \mathrm{~cm}^{-1} \mathrm{em}$ um equipamento Perkin-Elmer, modelo 1720. As fibras foram picadas em um moinho de facas, secas em estufa e prensadas em pastilhas de $\mathrm{KBr}$. Na faixa do espectro analisada, encontra-se a banda característica de grupamentos carbonila, relacionada aos hemiacetais dos carbonos anoméricos C1 (1636$1750 \mathrm{~cm}^{-1}$ ) e a banda de vibração axial da hidroxila (3300$3500 \mathrm{~cm}^{-1}$ ). Alterações observadas na grandeza nesses sinais permitem verificar a eficiência do tratamento de acetilação ${ }^{[16]}$.

A análise por microscopia eletrônica de varredura foi feita em um equipamento Zeiss DSM 960, usando-se elétrons secundários e voltagem de aceleração do feixe de elétrons entre $15-20 \mathrm{kV}$, em amostras recobertas com um liga condutora de Au-Pd.

\section{Resultados e Discussão}

A Figura 3 mostra imagens obtidas por MEV que evidenciam o aspecto superficial da bucha sem tratamento. Pode-se observar o entrelaçamento tridimensional das fibras, Fig.3a, e que, semelhantemente a outras fibras lignocelulósicas, a superfície das fibras de bucha é formada por uma arranjo regular de células parenquimáticas, Fig.3 $b^{[11,20]}$. Na Figura 4, está mostrado o aspecto da superfície após o tratamento de acetilação. Pode-se observar que a camada mais externa das fibras foi eliminada, revelando a estrutura fibrilar interna. Esse aspecto por si só mostra que o tratamento foi efetivo, pois, conforme observado ao se comparar as Figuras 3 e 4, 

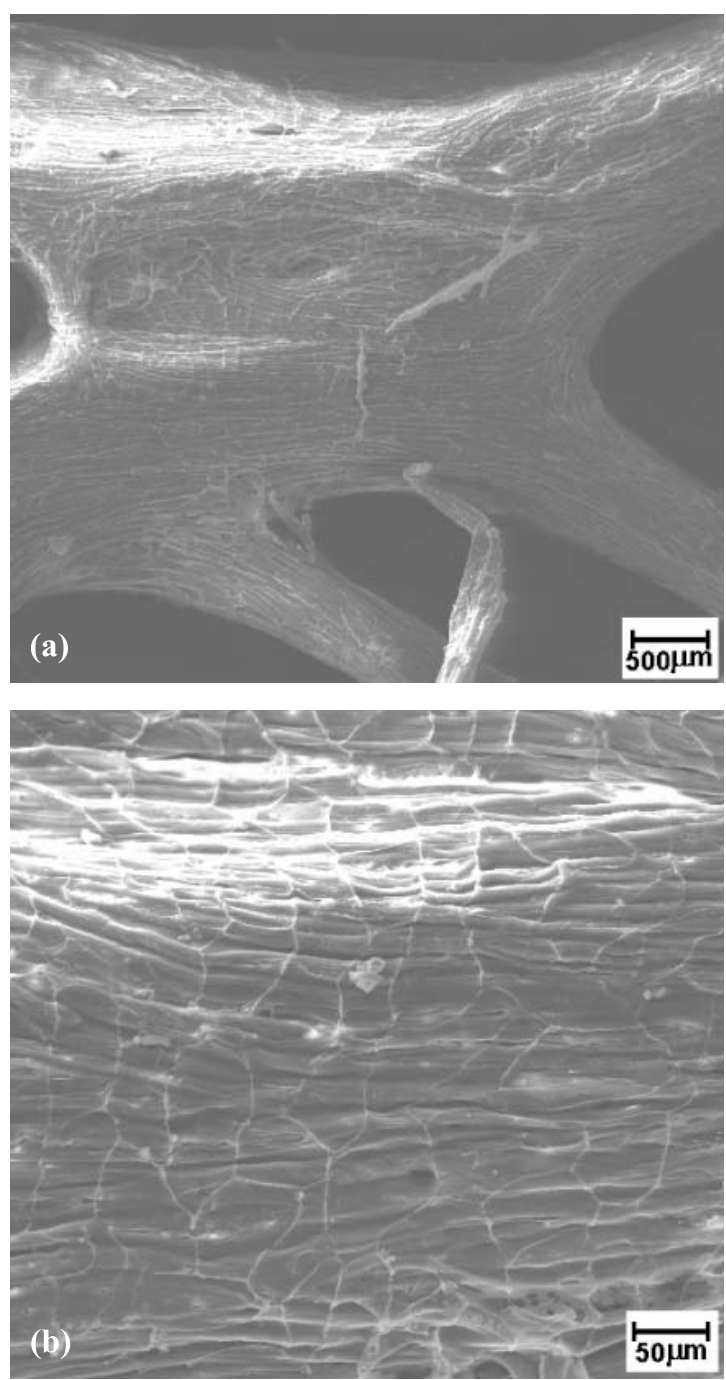

Figura 3. Fotomicrografia da bucha sem tratamento. (a) Arranjo tridimensional formando uma manta natural, $(b)$ detalhe da superfície.

parece que a área superficial da fibra aumentou, o que pode contribuir para aumentar a resistência da interface por acoplamento mecânico ${ }^{[21]}$.

A comprovação de que a reação de acetilação foi efetiva pode ser verificada comparando-se as Figuras 5 e 6, onde estão mostrados os espectros de infravermelho para a fibra natural (não acetilada), Fig.5, e acetilada, Fig.6. Pode-se observar no espectro da fibra não acetilada a presença de sinal em $3437 \mathrm{~cm}^{-1}$, característico da vibração axial das hidroxilas da celulose (hidroxilas dos carbonos 2, 3, e 6 da glucose) e um sinal em $1690 \mathrm{~cm}^{-1}$, atribuído ao grupo acetal, correspondente à carbonila das aldoses.

No espectro de infravermelho da fibra acetilada verificase uma redução significativa do sinal correspondente à hidroxila. A presença de grupamentos hidroxila na fibra tratada é comprovada pela presença do sinal em $3500 \mathrm{~cm}^{-1}$, referente a vibração axial das hidroxilas, o que indica que a acetilação não foi completa. Por outro lado, o sinal observado em $3640 \mathrm{~cm}^{-1}$ corresponde à grupamento $\mathrm{OH}$ de água adsorvida na fibra. $\mathrm{O}$ sinal intenso observado em $1722 \mathrm{~cm}^{-1}$
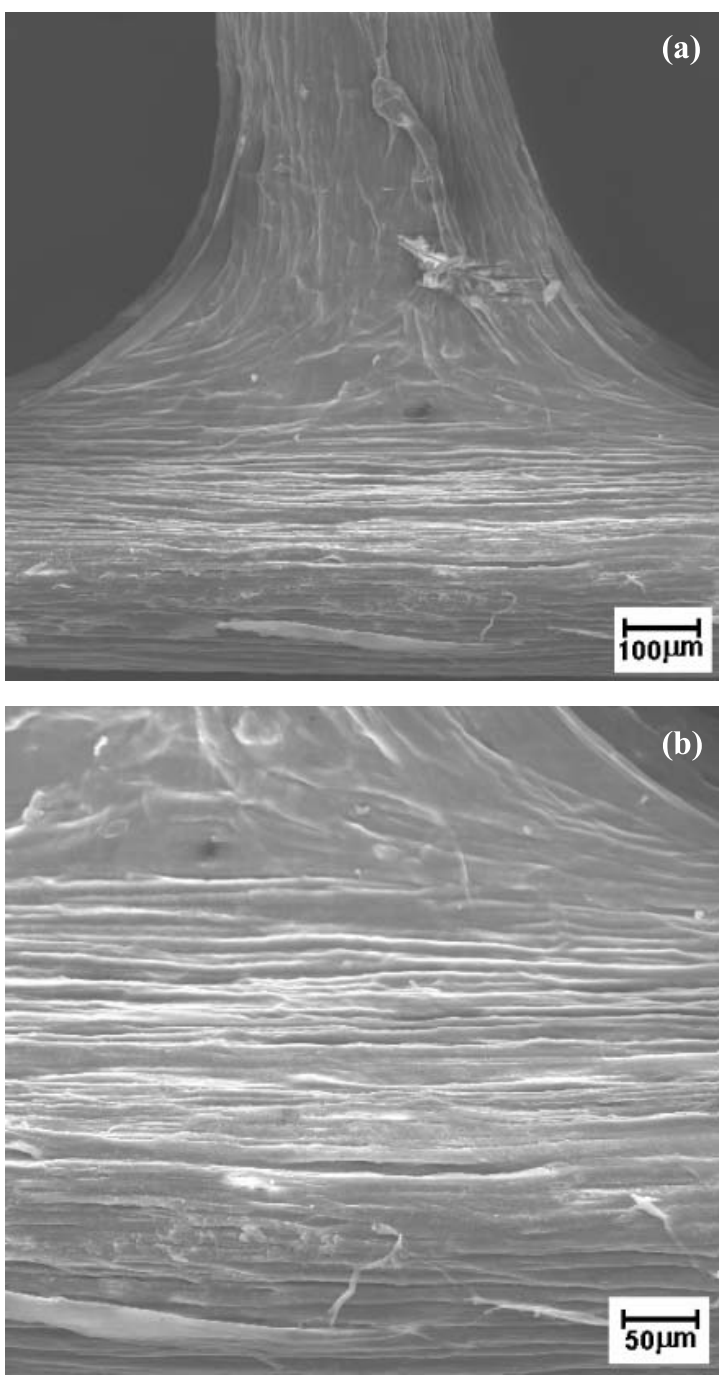

Figura 4. Fotomicrografia da bucha acetilada. (a) Aspecto geral mostrando a estrutura fibrilar interna. (b) Detalhe da superfície fibrilar observada após tratamento.

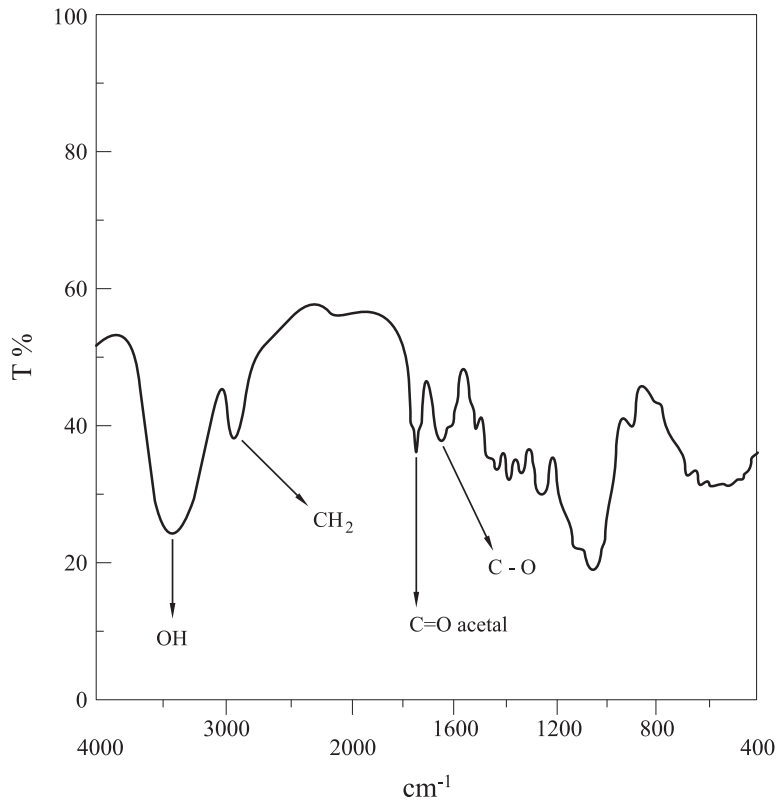

Figura 5. FTIR da fibra não acetilada. 


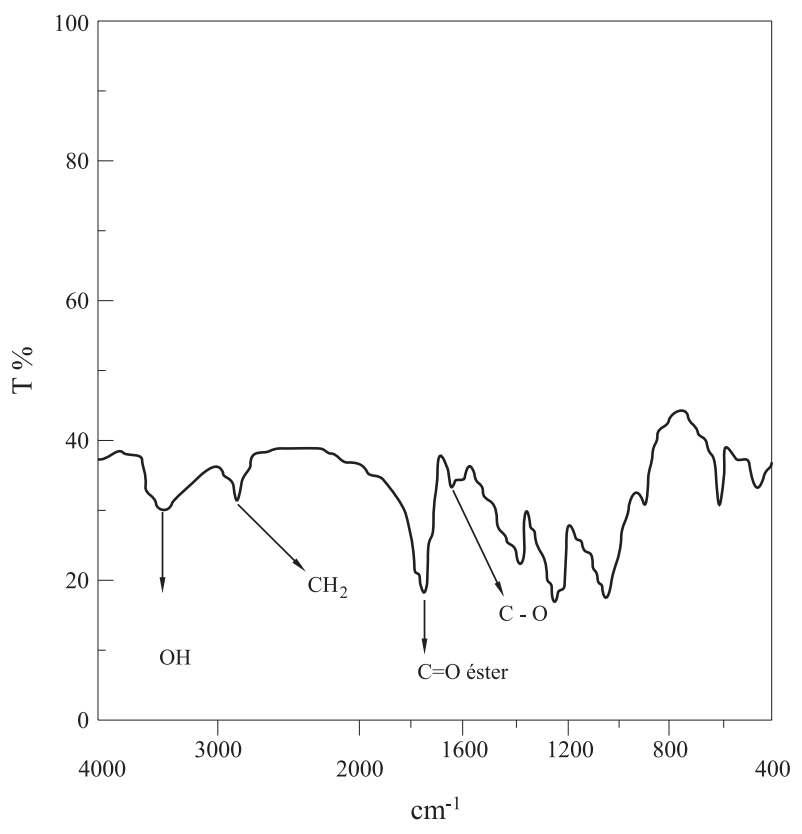

Figura 6. FTIR da fibra acetilada.

corresponde à carbonila de um éster (acetato), confirmando a ocorrência da reação de acetilação da fibra de Luffa cylíndrica.

Embora a esterificação dos grupo $\mathrm{OH}$ não tenha sido total, a reação parcial observada já é potencialmente interessante, pois reduz a higroscopicidade das fibras, visto que os sítios de hidroxila favorecem a absorção de umidade ${ }^{[19]}$. A verificação de que as fibras acetiladas têm menor higroscopicidade foi verificada pelos resultados da perda de massa abaixo de $100{ }^{\circ} \mathrm{C}$ em análise termogravimétrica ${ }^{[22]}$. Como conseqüência da redução de umidade, a estabilidade dimensional da fibra é aumentada, bem como sua resistência a ataques de microorganismos ${ }^{[19]}$.

\section{Conclusões}

Os resultados experimentais mostraram que houve uma reação parcial dos grupos hidroxila presentes na superfície da bucha. Conseqüentemente, a polaridade da fibra foi reduzida o que aumenta a compatibilidade com polímeros apolares. Os resultados obtidos indicam, portanto, a viabilidade do tratamento proposto.

\section{Agradecimentos}

Os autores agradecem ao CNPq pelo apoio financeiro.

\section{Referências Bibliográficas}

1. Schuh, T. G. \& Gayer, U. - “Automotive Applications of Natural Fiber Composites", in: Lignocellulosic-Plastics Composites, pp.181-195, Leão, A.L.; Carvalho, F.X.; Frollini, E. (eds.), Unesp publisher, Botucatu-São Paulo, Brazil (1997).

2. Schuh, T. G. - "Renewable Materials for Automotive Applications" in http://www.ienica.net/fibresseminar/schuh.pdf

3. Pejis, T. - Mat.Tech.\& Adv. Perf. Mat., 15, p.269 (2000).
4. Mohanty, A. K. \& Misra, M. - Polym.-Plast.Technol. \& Eng., 34, p.729 (1995).

5. Li, Y.; Mai, Y.-M. \& Ye, L. - Comp.Sci.\&Technol., 60, p.2037 (2000).

6. Coutts, R. S. P. - J. Mater. Sci. Letters, 9, p.1235 (1990).

7. Satyanarayana, K. G.; Sukumaran, K.; Kulkarni, A. G.; Pillai, S. G. K. \& Rohatgi, P. K. - Composites, 17, p.329 (1986).

8. d'Almeida, J. R. M. \& Monteiro, S. N. - “Compósitos reforçados por fibras naturais - oportunidades e desafios", in: Anais do 58․ Congresso Anual da Associação Brasileira de Metalurgia e Materiais, p.1725, Rio de Janeiro - RJ, julho (2003).

9. Paiva Júnior, C. Z.; de Carvalho, L. H.; Fonseca, V. M.; Monteiro, S. N. \& d'Almeida, J. R. M. - Polym.Testing, 23, p.131 (2004).

10. Aquino, R. C. M. P.; d'Almeida, J. R. M. \& Monteiro, S. N. J. Mater. Sci. Letters, 20, p.1017 (2001).

11. Boynard, C. A. \& d'Almeida, J. R. M. - Polym.-Plast. Technol. \& Eng., 39, p.489 (2000).

12. Boynard, C. A.; Monteiro, S. N. \& d'Almeida, J. R. M. - J. Appl. Polym. Sci., 87, p.1927 (2003).

13. Naglis, M. M. M. \& d'Almeida, J. R. M. - “Aspectos do Emprego de Fibras Naturais Como Reforço em Compósitos: Análise da Morfologia da Luffa Cilíndrica", in: Anais do 4은 MICROMAT, p.575, São Carlos - SP, out (1994).

14. Bledzki, A. K.; Reihmane, S. \& Gassan, J. - J. Appl. Polym. Sci., 59, p.1329 (1996)

15. Prasad, S. V.; Pavithram, C. \& Rohatgi, P. K. - J.Mater.Sci., 18, p.1443 (1983).

16. Calado, V.; Barreto, D. W. \& d'Almeida, J. R. M. - J. Mater. Sci.Letters, 19, p.2151 (2000).

17. Calado, V.; Barreto, D. W. \& d'Almeida, J. R. M. - Polym. \& Polym. Comp., 11, p.31 (2003).

18. Varma, I. K.; Krishan, S. R. A. \& Krishnamoorthy, S. Composites, 20, p.383 (1989).

19. Kumar, S. \& Agarwal, S. C. - "Chemical Modification of Wood with Thioacetic Acid", in: Graft Copolymerization of Lignocellulosic Fibers, cap.19, American Chemical Society, New York, (1982).

20. Mattoso, L. H. C.; Ferreira, F. C. \& Curvelo, A. A. S. - "Sisal Fiber: Morphology and Application in Polymer Composites", in: Lignocellulosic - Plastics Composites, pp.241-266, Leão, A.L.; Carvalho, F.X.; Frollini, E. (eds.), Unesp publisher, Botucatu-São Paulo, Brazil (1997).

21. Allen K. W. - Int. J. Adhesion \& Adhesives, 13, p.67 (1993).

22. d'Almeida, A. L. F. S.; Calado, V.; Barreto, D. W. \& d'Almeida, J.R.M. - "Análise termogravimétrica de fibras de Luffa cilindrica brutas e tratadas" in: Anais do XVI CBECIMAT, aceito para publicação, PortoAlegre - RS, dez (2004).

Enviado: 06/05/04

Reenviado: $30 / 11 / 04$

Aprovado: 07/12/04 